\title{
Targeting physical activity interventions for adults: When should intervention occur?
}

\author{
Katelyn M. Holliday ${ }^{\mathrm{a}, *}$, Dan Yu Lin ${ }^{\text {b }}$, Sujatro Chakladar ${ }^{\text {b }}$, Sheila F. Castañeda ${ }^{\text {c }}$, Martha L. Daviglus ${ }^{\text {d }}$, \\ Kelly R. Evenson ${ }^{\mathrm{a}}$, David X. Marquez ${ }^{\mathrm{e}}$, Qibin $\mathrm{Qi}^{\mathrm{f}}{ }$, Christina M. Shay ${ }^{\mathrm{g}}$, Daniela Sotres-Alvarez ${ }^{\mathrm{h}}$, Denise C. Vidot ${ }^{\mathrm{i}}$, \\ Donglin Zeng ${ }^{\mathrm{b}}$, Christy L. Avery ${ }^{\mathrm{a}}$ \\ a Department of Epidemiology, Gillings School of Global Public Health, University of North Carolina at Chapel Hill, 137 E. Franklin Street Suite 306, Chapel Hill, NC 27514, USA \\ b Department of Biostatistics, Gillings School of Global Public Health, University of North Carolina at Chapel Hill, 135 Dauer Drive, 3101 McGavran-Greenburg Hall, Chapel Hill, NC 27514, USA \\ c Graduate School of Public Health, San Diego State University, 5500 Campanile Dr., San Diego, CA 92182, USA \\ d Institute for Minority Health Research, University of Illinois at Chicago, 1819 W. Polk Street, Suite 246, Chicago, IL 60612, USA \\ e Department of Kinesiology and Nutrition, University of Illinois at Chicago, 1919 W. Taylor Street, Room 625, MC 994, Chicago, IL 60612, USA \\ ${ }^{\mathrm{f}}$ Department of Epidemiology and Population Health, Albert Einstein College of Medicine, 1300 Morris Park Avenue, Belfer 1306A, Bronx, NY 10461, USA \\ ${ }^{g}$ Department of Nutrition, University of North Carolina at Chapel Hill, 2201 McGavran- Greenberg Hall, Chapel Hill, NC 27599, USA \\ ${ }^{\text {h }}$ Collaborative Studies Coordinating Center, Department of Biostatistics, University of North Carolina at Chapel Hill, 137 E. Franklin Street, Suite 203, Chapel Hill, NC 27514, USA \\ ${ }^{\mathrm{i}}$ University of Miami, Miller School of Medicine, Clinical Research Building, 1120 NW 14th Street, Suite 1515, Miami, FL 33136, USA
}

\section{A R T I C L E I N F O}

\section{Article history:}

Received 1 September 2016

Received in revised form 29 November 2016

Accepted 21 December 2016

Available online 23 December 2016

\section{Keywords:}

Physical activity

Minority health

Population-based planning

\begin{abstract}
A B S T R A C T
Understanding demographic differences in transitions across physical activity (PA) levels is important for informing PA-promoting interventions, yet few studies have examined these transitions in contemporary multi-ethnic adult populations. We estimated age-, race/ethnicity-, and sex-specific 1-year net transition probabilities (NTPs) for National Health and Nutrition Examination Survey (2007-2012, n = 11,556) and Hispanic Community Health Study/Study of Latinos (2008-2011, $n=15,585)$ adult participants using novel Markovtype state transition models developed for cross-sectional data. Among populations with ideal PA ( $\geq 150 \mathrm{~min} /$ week; ranging from $56 \%$ (non-Hispanic black females) to $88 \%$ (non-Hispanic white males) at age 20), NTPs to intermediate PA ( $>0-<149 \mathrm{~min} /$ week) generally increased with age, particularly for non-Hispanic black females for whom a net $0.0 \%$ (95\% confidence interval $(\mathrm{CI}): 0.0,0.2)$ transitioned from ideal to intermediate PA at age 20 ; by age 70 , the NTP rose to $3.6 \%(95 \% \mathrm{CI}: 2.3,4.8)$. Heterogeneity in intermediate to poor ( 0 min/week) PA NTPs also was observed, with NTPs peaking at age 20 for Hispanic/Latino males and females [age $20 \mathrm{NTP}=3.7 \%$ (95\% CI: $2.0,5.5)$ for females and 5.0\% (1.2, 8.7) for males], but increasing throughout adulthood for non-Hispanic blacks and whites [e.g. age $70 \mathrm{NTP}=7.8 \%(95 \% \mathrm{CI}: 6.1,9.6 \%)$ for black females and $8.1 \%(4.7,11.6)$ for black males]. Demographic differences in PA net transitions across adulthood justify further development of tailored interventions. However, innovative efforts may be required for populations in which large proportions have already transitioned from ideal PA by early adulthood.
\end{abstract}

\section{Introduction}

Physical activity (PA) has been linked to prevention of a spectrum of chronic diseases as well as healthy longevity, compression of morbidity, quality of life maintenance, and reduced healthcare costs (USDHHS,

\footnotetext{
* Corresponding author at: 137 E. Franklin Street, Suite 306, Chapel Hill, NC 27514, USA. E-mail addresses: khausman@email.unc.edu (K.M. Holliday), lin@bios.unc.edu (D.Y. Lin), sujatro@live.unc.edu (S. Chakladar), scastaneda@mail.sdsu.edu (S.F. Castañeda), daviglus@uic.edu (M.L. Daviglus), kelly_evenson@unc.edu (K.R. Evenson), marquezd@uic.edu (D.X. Marquez), qibin.qi@einstein.yu.edu (Q. Qi), chris.shay@unc.edu (C.M. Shay),dsotres@email.unc.edu (D. Sotres-Alvarez), DVidot@med.miami.edu (D.C. Vidot), dzeng@bios.unc.edu (D. Zeng), christy_avery@unc.edu (C.L. Avery).
}

2008; WHO, 2009). Given its widespread benefits, the American Heart Association (AHA) included PA as a component of cardiovascular health, relying on the United States Department of Health and Human Services' 2008 Physical Activity Guidelines for Americans (USDHHS, 2008) to define levels of ideal, intermediate, and poor PA (Lloyd-Jones et al., 2010). For adults, ideal PA is at least 150 min of moderate PA per week, intermediate is any $\mathrm{PA}<150 \mathrm{~min} /$ week, and poor is no PA.

Nationally representative studies suggest that less than half of adults meet the definition of ideal PA, with $32 \%$ of adults classified as having poor PA (Lloyd-Jones et al., 2010). The prevalence of ideal PA is also known to decline with age and varies by sex and race/ethnicity (Shay et al., 2012). For example, women have a lower prevalence of ideal PA than men across all ages, with prevalence differences of $8 \%$ for ages 
20-64 that rise to 14\% after age 64 (Shay et al., 2012). Non-Hispanic white females report at least $10 \%$ higher prevalence of ideal PA than non-Hispanic black females across all ages (Shay et al., 2012). The prevalence of ideal PA is high [65.2\% (95\% CI: 64.0, 66.4)] among Hispanic/ Latino adults as compared with the overall United States adult population, but varies by background with those of Cuban background having the lowest proportion of ideal PA (González et al., 2016).

Given the high prevalence of poor PA, understanding sex- and race/ ethnicity-specific transition patterns between levels of PA, including the ages associated with accelerated loss of ideal PA, would be useful for planning and targeting PA interventions. Yet, few studies have evaluated PA transitions across the life course, reflecting difficulties of conducting longitudinal studies of PA with comparable assessment protocols in multi-ethnic populations spanning relevant age ranges. Fortunately, recent advances in statistical methods have enabled the evaluation of PA net transition probabilities using contemporary crosssectional data (van de Kassteele et al., 2012), which provide important population-level information on the age-specific net increases or decreases in the proportion of the population within a specific PA category across adulthood. We therefore estimated age-, race/ethnicity-, and sexspecific PA net transition probabilities for non-Hispanic white, non-Hispanic black, and Hispanic/Latino adults to identify the ages associated with changes in PA.

\section{Methods}

We estimated PA net transitions using cross-sectional data from two population-based studies: the National Health and Nutrition Examination Survey (NHANES) (CDC, 2014) and the Hispanic Community Health Study/Study of Latinos (HCHS/SOL) (Lavange et al., 2010; Sorlie et al., 2010). Given the large difference in PA recommendations for children and adults, the analysis was restricted to adults (age $\geq 20$ ) in these populations.

NHANES is a complex, multistage probability sample of the civilian, noninstitutionalized United States population (CDC, 2013). We combined data from three recent continuous NHANES cycles (2007-2008, 2009-2010, and 2011-2012) for non-Hispanic black ( $\mathrm{n}=3797)$ and non-Hispanic white $(\mathrm{n}=7759)$ adults at least 20 years of age.

$\mathrm{HCHS} / \mathrm{SOL}$ is a multi-center, community-based cohort study of $\mathrm{n}=$ 16,415 self-identified Hispanic/Latinos aged 18-74 years at baseline (2008-2011) selected through a stratified two-stage area probability sample of household addresses (Lavange et al., 2010; Sorlie et al., 2010). Although NHANES over-sampled Mexican-Americans, we chose to focus on HCHS/SOL ( $n=15,585$ ) due to its large communitybased sample of diverse Hispanic/Latino backgrounds, facilitating examination of potentially important differences in PA behaviors among subgroups.

AHA defined an ideal adult PA level as meeting the 2008 Physical Activity Guidelines for Americans for moderate-to-vigorous aerobic PA ( $\geq 3$ metabolic equivalents) (2008), an intermediate level as completing some PA but not enough to meet guidelines, and a poor level as participating in no PA (Table S1) (Lloyd-Jones et al., 2010). Although NHANES and HCHS/SOL have both self-reported and accelerometry derived PA (Arredondo et al., 2016; Schuna et al., 2013), we used self-reported data to ensure comparability with the guidelines, which were based on self-reported data (see Appendix, Supplemental Methods for further PA measurement and classification details).

\subsection{Statistical analyses}

We used cross-sectional data from NHANES and HCHS/SOL baseline exam along with net transition probability models proposed by van de Kassteele et al. (2012) to estimate age-, race/ethnicity-, and sex-specific net transition probabilities between ideal, intermediate, and poor PA for non-Hispanic blacks, non-Hispanic whites, and Hispanic/Latinos (both overall and by country of origin). These net transition probabilities reflect the net flow of the population between PA categories at a given age, thereby allowing use of cross-sectional as opposed to longitudinal data in calculations. For example, in a cohort of 100 20-year-olds with ideal PA, if 40 individuals transition from ideal to intermediate PA by age 21 while 15 individuals with intermediate PA transition to ideal PA by age 21 , a net $40-15=25$ moved from ideal to intermediate PA at the population level. Thus, the net transition probability is expressed as this net number divided by the initial population with ideal PA, or 25/ $100=0.25$. In turn, the net flow for intermediate to ideal PA is 0 since more individuals moved from ideal to intermediate PA than vice versa, making the net transition probability 0 .

In order to estimate net transition probabilities, we first estimated predicted smoothed age-, race/ethnicity-, and sex-specific prevalences of each PA level (van de Kassteele et al., 2012) using a multinomial logit model (Hosmer and Lemeshow, 2000; McCullagh and Nelder, 1989) and P-spline smoothing (Eilers and Marx, 1996), accounting for genetically-determined first degree relative family structure (HCHS/ SOL) and complex sampling designs (HCHS/SOL and NHANES) with cluster sampling methods. Smoothing was implemented to lessen the influence of implausible increases or decreases in the prevalence of ideal, intermediate, and poor PA between consecutive ages (Fig. S1). Details of net transition probability estimation are available (see Appendix Supplemental Methods). Finally, we estimated 95\% confidence intervals using bootstrapping (1000 samples), wherein the age-specific prevalence was simulated from its asymptotic distribution and net transition probabilities were then computed for each simulated prevalence.

We performed several analyses to examine two model assumptions, namely the stability of net transition probabilities over one-year intervals and the influence of the cost matrix. The first analysis supported the assumption of stability (Figs. S2/S3), showing for example that the predicted prevalence of ideal, intermediate, and poor PA for 20092010 and 2011-2012 calculated using net transition probabilities based on the 2007-2008 NHANES cycle closely aligned with the observed prevalence in those years. Additionally, sensitivity analyses imposing a range of cost constraints (Table S2) showed little effect on the estimated net transition probabilities (Figs. S4/S5). Therefore, main results are presented using the default cost matrix suggested by van de Kassteele.

Finally, we extrapolated our results to the non-Hispanic black and white average 2007-2012 civilian, non-institutionalized United States population and to the 2010 Hispanic/Latino population within HCHS/ SOL community census tracts to estimate the net number of individuals transitioning between PA levels. Extrapolation was accomplished by multiplying the age, sex-, and race/ethnicity-specific net transition probabilities (e.g., ideal to intermediate) by the prevalence of the initial PA level (e.g., ideal) and the appropriate age-, race/ethnicity-, and sexspecific population sizes. Statistical analyses were primarily performed in 2015 using SAS (Cary, NC) with some steps completed in R (Vienna, Austria; packages lpSolve, MASS, Graphics, and Matrix) (calculating supply and demand inputs) and STATA (College Station, TX) (Table 1 weighted estimates for HCHS/SOL). This study was approved by local Institutional Review Boards.

\section{Results}

All race/ethnicity- and sex-specific populations had a median age in the fourth decade of life and median body mass index (BMI) classified as overweight or obese (BMI $\geq 25 \mathrm{~kg} / \mathrm{m}^{2}$ ) (Table 1 ). The prevalence of ideal PA across adulthood ranged from a low of $47.3 \%$ among non-Hispanic black females to a high of $75.3 \%$ among Hispanic/Latino males. Yet at age 20, the age-specific prevalence of ideal PA was similar among males of all race/ethnicity groups (non-Hispanic black: $82.2 \%$ (95\% CI: 76.6, 87.7), non-Hispanic white: $87.7 \%$ (84.5, 90.9), Hispanic/Latinos: $87.3 \%(84.5,90.0)$ ) but varied among females (non-Hispanic black: 56.0\% (49.2, 62.9), non-Hispanic white: 74.3\% (69.3, 79.3), Hispanic/Latinos: $69.9 \%(65.7,74.1))$. Across adulthood, $17 \%$ to $35 \%$ of race/ 
Table 1

Cross-sectional demographics of $\mathrm{N}=27,141$ participants $\geq 20$ years in the 2007-2012 NHANES ${ }^{\mathrm{a}}$ and the 2008-2011 $\mathrm{HCHS}_{\text {SOL }}^{\mathrm{b}}$.

\begin{tabular}{|c|c|c|c|c|c|c|}
\hline \multirow[t]{2}{*}{ Characteristic } & \multicolumn{2}{|c|}{ Non-Hispanic black ${ }^{\mathrm{a}}$} & \multicolumn{2}{|c|}{ Non-Hispanic white ${ }^{\mathrm{a}}$} & \multicolumn{2}{|l|}{ Hispanic|Latino $^{\mathrm{b}}$} \\
\hline & Female & Male & Female & Male & Female & Male \\
\hline $\mathrm{N}$ & 1962 & 1835 & 3896 & 3863 & 9393 & 6192 \\
\hline Median age (IQR) & $44(32-56)$ & $43(30-55)$ & $49(36-63)$ & $48(35-60)$ & $42(31-54)$ & $41(30-52)$ \\
\hline \multicolumn{7}{|l|}{ Physical activity } \\
\hline Ideal (\%) & 47.3 & 67.1 & 58.9 & 73.2 & 58.7 & 75.3 \\
\hline Intermediate (\%) & 17.5 & 10.5 & 16.3 & 9.6 & 15.4 & 9.2 \\
\hline Poor (\%) & 35.2 & 22.3 & 24.9 & 17.2 & 25.9 & 15.5 \\
\hline Missing (N) & 3 & 4 & 6 & 13 & 152 & 122 \\
\hline Median BMI (IQR) & $30.9(26.0-36.3)$ & $27.9(23.9-32.7)$ & $26.9(23.2-32.0)$ & $27.8(24.8-31.5)$ & $29.0(25.5-33.5)$ & $28.4(25.6-31.8)$ \\
\hline
\end{tabular}

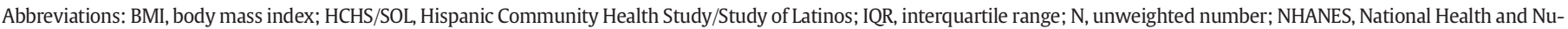
trition Examination Survey.

a Adjusted for sampling weights (except for $\mathrm{N}$ ) to represent non-institutionalized, civilian, resident United States non-Hispanic blacks and non-Hispanic whites.

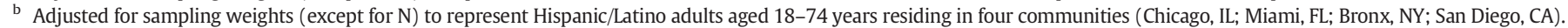

ethnicity- and sex-specific groups reported no PA during leisure, work, or transportation, placing them in the poor PA category.

A similar pattern of increasing ideal to intermediate net transition probabilities with age was observed for non-Hispanic blacks and nonHispanic whites, although differences became more pronounced by mid to late adulthood (Fig. 1, Table S3). For example, a net 0.0\% (95\% CI: $0.0,0.2 \%$ ) of non-Hispanic black females aged 20 transitioned from ideal to intermediate PA one year later. By age 70 , this net transition probability increased to $3.6 \%$ ( $95 \% \mathrm{CI}: 2.3,4.8$ ) as compared with $2.4 \%$ $(1.8,3.0)$ for non-Hispanic white females (Table S3). Similarly, non-Hispanic black males experienced a low $0.3 \%(95 \% \mathrm{CI}: 0.0,0.7)$ ideal to intermediate net transition probability at age 20 , but this net transition probability rose to a net $3.0 \%(2.0,4.0)$ by age 70 as compared with $1.6 \%$ (95\% CI: 1.1, 2.1) for non-Hispanic white males (Table S3).

In contrast to the pattern of increasing ideal to intermediate net transition probabilities with age estimated among non-Hispanic blacks and non-Hispanic whites, Hispanic/Latino males and females experienced minimal increases in the ideal to intermediate net transition probability with age. Indeed, a net $0.7 \%(95 \% \mathrm{CI}: 0.3,1.2)$ of Hispanic/Latino females aged 20 with ideal PA transitioned to intermediate PA by age 21 and a similar $0.6 \%$ (95\% CI: 0.0, 1.5) of Hispanic/Latino females aged 70 with ideal PA transitioned to intermediate by age 71 (Fig. 1, Table S3). Despite these overall differences from non-Hispanic blacks and whites, HCHS/SOL participants displayed heterogeneity by country of origin, with females from the Dominican Republic and South America and males from Cuba, Puerto Rico, and South America experiencing patterns more similar to non-Hispanic whites and blacks (Figs. S6 and S7).

We also observed population-specific patterning in net transition probabilities from intermediate to poor PA (Fig. 2, Table S3). Non-Hispanic black and non-Hispanic white females experienced little net movement between intermediate and poor PA levels during young adulthood, but intermediate to poor net transition probabilities increased beginning at approximately age 30 , reaching a maximum at 70 years of age (e.g., 7.8\% (95\% Cl: 6.1, 9.6) for non-Hispanic black females) (Table S3). Non-Hispanic black and non-Hispanic white males experienced a similar pattern of increasing intermediate to poor PA net transition probabilities with age; however, males of both race/ethnicity groups had higher intermediate to poor PA net transition probabilities during young adulthood than their female counterparts, [e.g., $2.9 \%(95 \%$ CI: $0.4,5.4)$ for non-Hispanic white males vs. 0.0\% (95\% CI: $0.0,0.6$ ) for non-Hispanic white females at age 20] (Fig. 2, Table S3), although results for males were notably less precise. In contrast, Hispanic/ Latino males and females began with higher intermediate to poor net transition probabilities in young adulthood [e.g., 3.7\% (95\% CI: 2.0, 5.5) for females at age 20 ] that decreased with age [e.g., to $0.0 \%(95 \% \mathrm{CI}$ : $0.0,1.2$ ) for females at age 70] (Fig. 2, Table S3). Similar to non-Hispanic black and non-Hispanic white males, Hispanic/Latino males also had higher intermediate to poor net transition probabilities earlier in
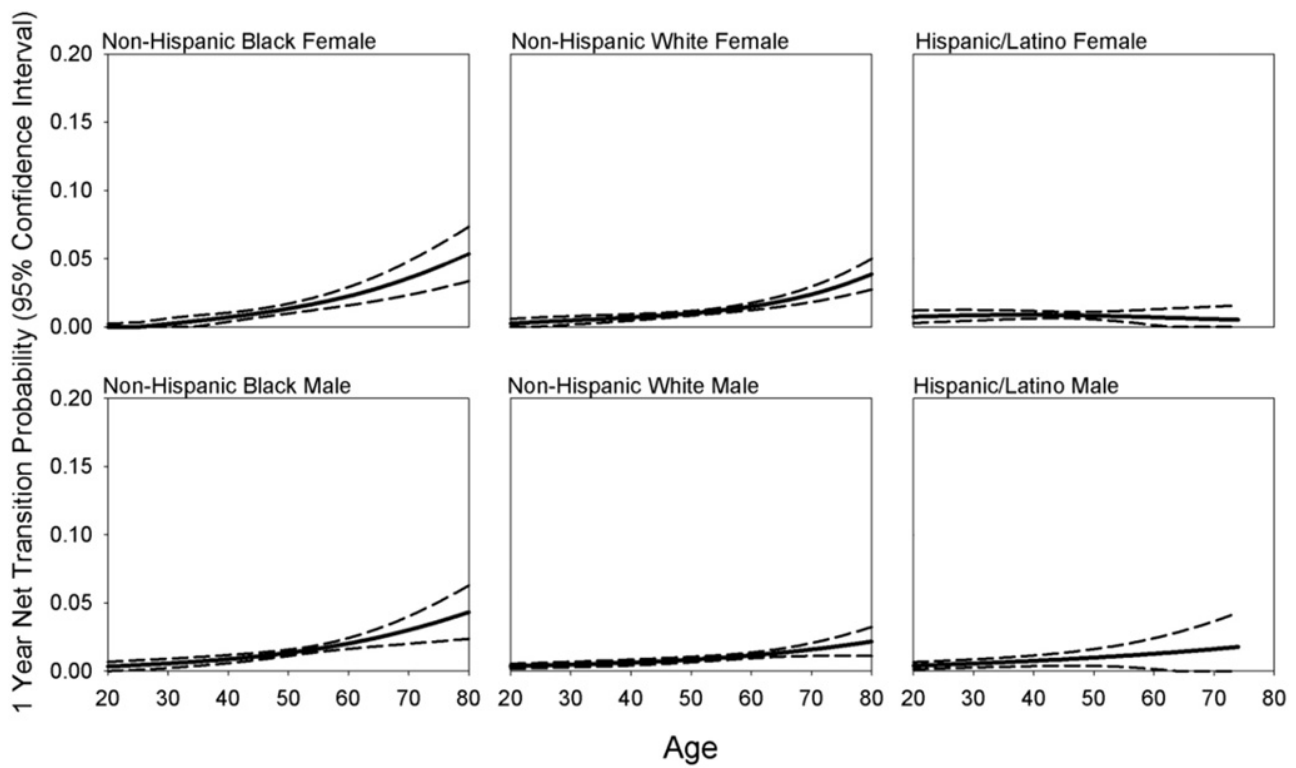

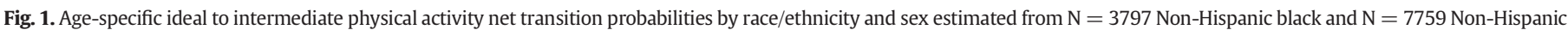
white 2007-2012 NHANES participants and N = 15,585 Hispanic/Latino 2008-2011 HCHS/SOL participants. 

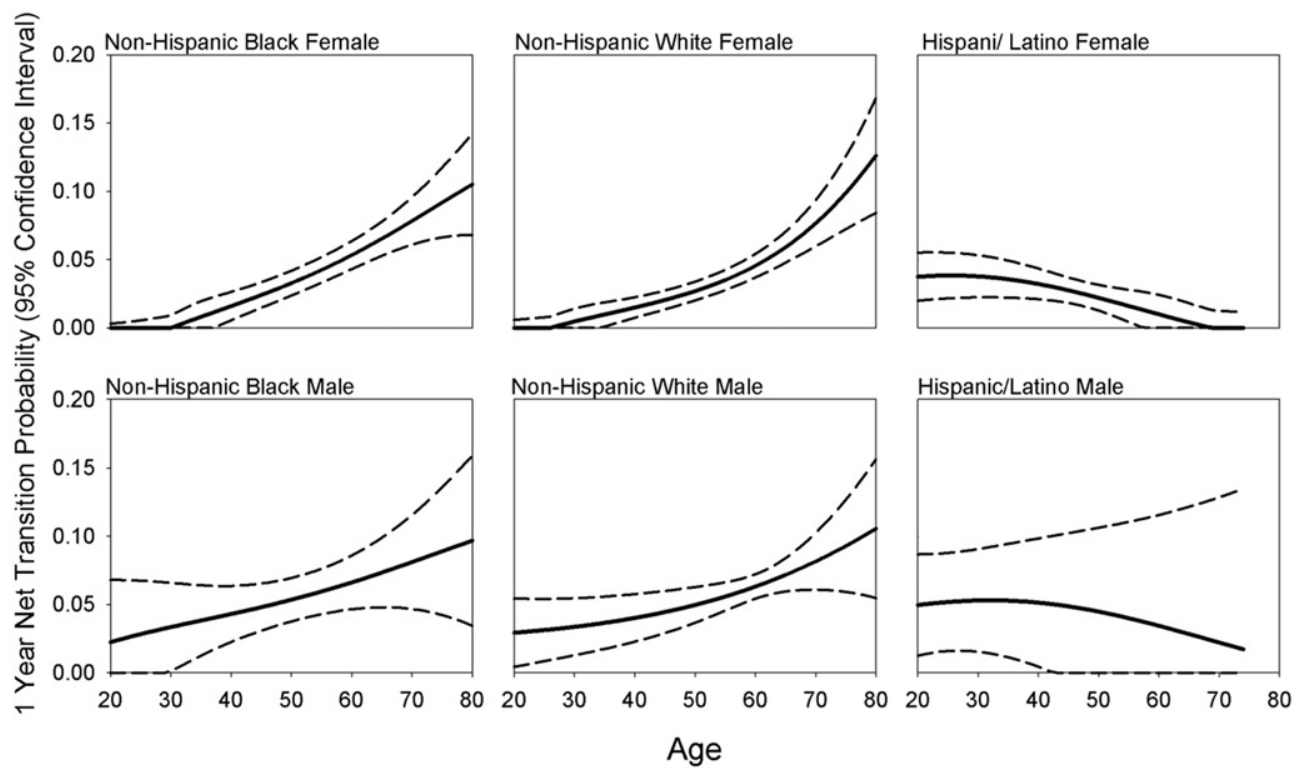

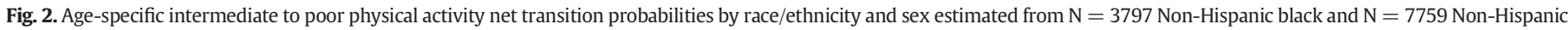
white 2007-2012 NHANES participants and N = 15,585 Hispanic/Latino 2008-2011 HCHS/SOL participants.

adulthood than their female counterparts [e.g., Hispanic/Latino females 3.7\% (95\% CI: 2.0, 5.5) versus Hispanic/Latino males 5.0\% (95\% CI: 1.2, 8.7) at age 40], although results were again considerably less precise for males (Table S3).

We extrapolated to the civilian, non-institutionalized United States' population to contrast the net transition probability by age with the net number of people transitioning to intermediate and poor PA by age and sex for non-Hispanic blacks and non-Hispanic whites. In contrast with the net transition probabilities, which increased with age, the net number of individuals transitioning between ideal and intermediate PA peaked in middle-age for non-Hispanic blacks and non-Hispanic whites, with net numbers peaking approximately 5 years earlier for non-Hispanic blacks and 6 years earlier for males (i.e., non-Hispanic black females peaked at age 55, non-Hispanic black males at age 49 , nonHispanic white females peaked at age 61 , and non-Hispanic white males at age 54) (Fig. 3). A similar pattern emerged for the net transitions from intermediate to poor PA (Fig. 3), with the net number transitioning from intermediate to poor PA peaking approximately 5 years after the peak ideal to intermediate PA net number for most sex- and race/ethnicity-specific groups.

In contrast, the greatest net numbers of Hispanic/Latinos in the four HCHS/SOL communities transitioned between ideal and intermediate PA at younger ages than did non-Hispanic blacks or whites (Fig. S8). Likewise, the net numbers of Hispanic/Latinos transitioning between intermediate and poor PA peaked at younger ages than non-Hispanic blacks or whites (age 44 for Hispanic/Latino males and age 26 for Hispanic/Latino females) (Fig. S9).

\section{Discussion}

Our study used methods proposed by van de Kassteele et al. (2012) with contemporary, routinely collected data to identify sex and racial/ ethnic differences in the patterning of PA net transition probabilities throughout adulthood. This method overcomes difficulties of longitudinal data collection and the potential for cohort effects to influence transitions calculated from longitudinal data (Anderssen et al., 1996). Patterns of PA net transition probabilities were similar among non-Hispanic blacks and whites, exhibiting a general trend of increasing ideal to intermediate and intermediate to poor net transition probabilities with age, although the magnitude varied by group. However, Hispanic/Latinos experienced a very different pattern, particularly for intermediate to poor transitions, which were high in young adulthood but diminished with age.

Given current low levels of ideal PA among adults (Lloyd-Jones et al., 2010), examination of PA trends throughout adulthood is warranted. Although calculating individual-level transitions over the life course is possible with decades of longitudinal research, this is expensive, timeconsuming, and burdensome for participants as well as potentially biased due to changing PA measurement techniques over time. Most existing studies of longitudinal patterns of adult PA evaluated short timespans, which do not allow consideration of changes across the entire age spectrum. For example, the Australian Longitudinal Study on Women's Health followed a cohort of middle aged (45-50 at baseline) and older women (70-75 at baseline) and reported longitudinal changes in self-reported PA over four years (Brown et al., 2007). This study
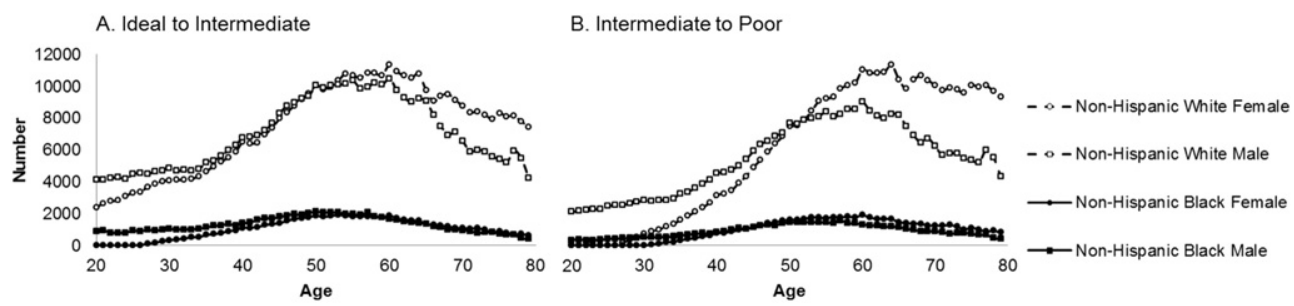

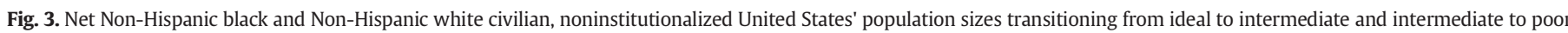
physical activity in one year. 
found that $34 \%$ and $23 \%$ of middle-aged and older women, respectively, maintained ideal PA over all 4 years while $26 \%$ and $17 \%$ increased their PA and 18\% and 26\% decreased PA. In the Coronary Artery Risk Development in Young Adults Study (CARDIA), seven-year changes in self-reported PA were observed among those aged 18-30 at baseline, although the measure of PA used is not comparable to the AHA definitions of ideal, intermediate, and poor PA (Anderssen et al., 1996). Nonetheless, the CARDIA results suggested that while PA score tracked moderately over the seven years of follow-up, substantial decreases in PA were observed in this young adult population.

When extrapolated to the United States' population, the peak net loss of individuals from more ideal PA states occurs earlier in adulthood for non-Hispanic blacks and males than their non-Hispanic white and female counterparts. While this might be expected for males, who have a higher prevalence of ideal PA at younger ages and thus a larger proportion of the population eligible to transition away from ideal PA than females, non-Hispanic blacks already begin with lower prevalence of ideal PA than non-Hispanic whites in young adulthood. Although this study did not examine the effects of participant-specific PA trajectories on health outcomes, the observed low prevalence of ideal PA in young non-Hispanic blacks combined with earlier peak net loss of ideal PA place non-Hispanic blacks at risk of extended exposure to inadequate PA, potentially contributing to heath disparities experienced by this population. Ultimately, non-Hispanic black females, who both start with the lowest prevalence of ideal PA at age 20 and attain the highest ideal to intermediate PA net transition probability, seem to be at greatest need of targeted interventions for initiating and maintaining ideal PA. Future studies may examine how demographic-specific PA trajectories relate to health outcomes, potentially providing information on when demographically-targeted PA interventions should be implemented to reduce specific negative health outcomes.

The Community Preventative Services Task Force (CPSTF) recommends several categories of evidence-based PA interventions including social-behavioral, informational, and environmental/policy approaches (CPSTF, 2014; Kahn et al., 2002). Several specific types of interventions were considered effective at increasing PA including point of decision prompts, community-wide campaigns, social support in communities, individually-adapted health behavior change strategies, and enhanced access to places for PA combined with informational outreach activities (CPSTF, 2014; Kahn et al., 2002). In all cases, the CPSTF indicated that these specific types of interventions are thought to be effective in diverse populations as long as the intervention is tailored to a specific target population. In some cases, the suggestion of tailoring was based on demonstrated effectiveness in studies of specific demographic populations and in others by evidence that generic interventions applied to heterogeneous groups became effective only after they were tailored.

Understanding the age-, race/ethnicity-, and sex-specific patterning of changes in PA can therefore be combined with CPSTF recommendations to identify specific subgroups in need of tailored interventions. For example, our results suggest that non-Hispanic blacks may be in particular need of interventions tailored to maintain ideal PA across the life course. On the other hand, Hispanic/Latino females may need tailored interventions to reduce movement between intermediate and poor PA during young adulthood. While non-Hispanic blacks and whites of both sexes may benefit from interventions that reduce movement from intermediate to poor PA, males would particularly benefit from interventions during young-adulthood when their net transition probabilities are greater than those for females. All age-, race/ethnicity-, and sex- specific groups need interventions designed to improve poor PA, suggesting that interventions targeted not only at traditional demographic groups but also at those with specific levels of PA would be appropriate. Further, the high burden of non-ideal PA at the population level may be difficult to impact with currently developed interventions reviewed and recommended by CPSTF, many of which can only be implemented on a small scale. Therefore, innovative interventions may need to be developed to attain population-level improvement in PA.

\subsection{Study limitations}

Although the method used in this study is innovative and has several strengths, limitations should be discussed. First, using the AHA definitions to classify PA complicates the ability to understand PA net transitions across the entire life course given differences in recommendations for adults and children. Second, classification of PA relied on self-reported PA, which has known limitations of recall and social-desirability bias. We focused on self-reported measures as opposed to accelerometry data as the guidelines on which PA classification was based were established using self-reported data, and these guidelines are not well-suited for PA derived from accelerometry (Thompson et al., 2016). While our results for non-Hispanic blacks and whites are generalizable to the civilian, non-institutionalized United States' population, our results for Hispanic/Latinos are generalizable to the four HCHS/ SOL communities. Nevertheless, the HCHS/SOL study allowed us to examine net transition probabilities among groups with differing countries of origin, capturing potentially important behavior differences in Hispanic/Latino populations. Finally, calculation of net transition probabilities relied on the assumption of stability of the net transition probabilities over time, limiting estimation to one-year net transitions. Longer net transitions would require use of longitudinal data or stronger, untested assumptions (e.g., that net transition probabilities remained stable across longer periods).

Some of the largest differences in PA net transition probability patterns were observed between Hispanic/Latinos (data provided by the HCHS/SOL study) and non-Hispanic blacks and whites (data provided by the NHANES), potentially suggesting that race/ethnic-specific differences were instead reflective of study-specific differences. Yet, previous research in other cohorts (Joseph et al., 2016; Willey et al., 2010; Hsia et al., 2005) has demonstrated differences in PA patterning in adulthood by Hispanic/Latino ethnicity, and HCHS/SOL investigators used PA questionnaires that were nearly identical to NHANES questionnaires, reducing the potential for systematic study-specific differences in PA assessment. Further, leveraging data from the HCHS/SOL allowed us to expand inference in Hispanic/Latinos to include several subgroups poorly represented in NHANES (e.g. Cubans, Puerto Ricans, Dominicans, and South Americans), potentially increasing generalizability of study findings to the broader Hispanic/Latino population.

\section{Conclusions}

Ultimately, this study suggests age-, race/ethnicity, and sex-specific patterning of the loss in ideal and intermediate PA across adulthood, with several important groups identified for targeted interventions at different ages. Non-Hispanic blacks and males experienced peak population shifts at least five years earlier in life than did non-Hispanic whites and females, respectively; however non-Hispanic black females were identified as the group potentially in greatest need of PA interventions. Hispanic/Latinos experienced very different net transition patterns from non-Hispanic blacks and whites, with the largest net transition probabilities found early in adulthood instead of increasing with age. Finally, all groups may be in need of innovative interventions to reduce population level burden of poor PA.

\section{Conflicts of interest statement}

The authors declare that there are no conflicts of interest.

\section{Funding}

This work was supported by the National Heart, Lung, and Blood Institute (NHLBI) (R21HL121580 and R00HL098458). The Hispanic Community Health Study/Study of Latinos was carried out as a collaborative study supported by contracts from the NHLBI to the University of North Carolina (N01-HC65233), University of Miami (N01-HC65234), Albert 
Einstein College of Medicine (N01-HC65235), Northwestern University (N01-HC65236), and San Diego State University (N01-HC65237). The following Institutes/Centers/Offices contribute to the HCHS/SOL through a transfer of funds to the NHLBI: National Institute on Minority Health and Health Disparities, National Institute on Deafness and Other Communication Disorders, National Institute of Dental and Craniofacial Research, National Institute of Diabetes and Digestive and Kidney Diseases, National Institute of Neurological Disorders and Stroke, NIH Institution-Office of Dietary Supplements. Dr. Qi is supported by a Scientist Development Award (K01HL129892) from the NHLBI. KMH has been supported by an NHLBI training grant (T32- HL007055).

\section{Acknowledgements}

The authors thank the staff and participants of HCHS/SOL and NHANES for their important contributions (Investigators website http://www.cscc.unc.edu/hchs/). This article has not been published elsewhere.

\section{Appendix A. Supplementary data}

Supplementary data to this article can be found online at http://dx. doi.org/10.1016/j.ypmed.2016.12.036.

\section{References}

Anderssen, N.J., Jacobs, D.R., Sidney, S., Bild, D.E., Stemfeld, B., Slattery, M.L., Hannan, P., 1996. Change and secular trends in physical activity patterns in young adults: a seven-year longitudinal follow-up in the coronary artery risk development in young adults study. Am. J. Epidemiol. 143, 351-362.

Arredondo, E., Sotres-Alvarez, D., Stoutenberg, M., Davis, S., Crespo, N., 2016. Physical activity levels in U.S. Latino/Hispanic adults: results from the Hispanic community health study/study of Latinos. Am. J. Prev. Med. 50, 500-508.

Brown, W.J., Burton, N.W., Heesch, K.C., 2007. Physical Activity and Health in Mid Age and Older Women. The Office for Women, Department of Families, Community Services, and Indigenous Affairs, Canberra.

CDC, 2013. Centers for Disease Control and Prevention/National Center for Health Statistics Continuous NHANES Web Tutorial. Sample Design. http://www.cdc.gov/nchs/ tutorials/nhanes/surveydesign/SampleDesign/intro.htm (Accessed June 5, 2014).

CDC, 2014. Centers for Disease Control and Prevention/National Center for Health Statistics National Heath and Nutrition Examination Survey. http://www.cdc.gov/nchs/ nhanes/about_nhanes.htm (Accessed June 5, 2014).

CPSTF, 2014. The Community Preventive Services Task Force Behavioral and Social Approaches to Increase Physical Activity: Individually-adapted Health Behavior Change Programs. http://www.thecommunityguide.org/pa/behavioral-social/ individuallyadapted.html (Accessed January 7, 2016).

Eilers, P.H.C., Marx, B.D., 1996. Flexible smoothing with B-splines and penalties (with discussion). Stat. Sci. 11, 89-121.
González, H.M., Tarraf, W., Rodriguez, C.J., Gallo, L.C., Sacco, R.L., Talavera, G.A., Heiss, G., Kizer, J.R., Hernandez, R., Davis, S., Schneiderman, N., Daviglus, M.L., Kaplan, R.C., 2016. Cardiovascular health among diverse Hispanics/Latinos: Hispanic Community Health Study/Study of Latinos (HCHS/SOL) results. Am. Heart J. 176, 134-144.

Hosmer, D.W., Lemeshow, S., 2000. Applied Logistic Regression. second ed. Wiley, New York.

Hsia, J., Wu, L.L., Allen, C., Oberman, A., Lawson, W.E., Torrens, J., Safford, M., Limacher, M.C., Howard, B.V., 2005. Physical activity and diabetes risk in postmenopausal women. Am. J. Prev. Med. 28 (1), 19-25.

Joseph, J.J., Echouffo-Tcheugui, J.B., Golden, S.H., Chen, H., Swords Jenny, S., Carnethon, M.R., Jacobs, D., Burke, G.L., Vaidya, D., Ouyang, P., Bertoni, A.G., 2016. Physical activity, sedentary behaviors and the incidence of type 2 diabetes mellitus: the Multi-Ethnic Study of Atherosclerosis (MESA). BMJ Open Diabetes Res. Care 4, e000185. http:// dx.doi.org/10.1136/bmjdrc-2015-000185.

Kahn, E.B., Ramsey, L.T., Brownson, R.C., Heath, G.W., Howze, E.H., Powell, K.E., Stone, E.J. Rajab, M.W., Corso, P., 2002. The effectiveness of interventions to increase physical activity: a systematic review. Am. J. Prev. Med. 22, 73-107.

Lavange, L.M., Kalsbeek, W.D., Sorlie, P.D., Aviles-Santa, L.M., Kaplan, R.C., Barnhart, J., Liu, K., Giachello, A., Lee, D.J., Ryan, J., Criqui, M.H., Elder, J.P., 2010. Sample design and cohort selection in the Hispanic Community Health Study/Study of Latinos. Ann. Epidemiol. 20, 642-649.

Lloyd-Jones, D.M., Hong, Y., Labarthe, D., Mozaffarian, D., Appel, L.J., Van Horn, L., Greenlund, K., Daniels, S., Nichol, G., et al., 2010. Defining and setting national goals for cardiovascular health promotion and disease reduction: the American Heart Association's strategic impact goal through 2020 and beyond. Circulation 12, 1586-1613.

McCullagh, P., Nelder, J., 1989. Generalized Linear Models. second ed. Chapman and Hall/ CRC, London.

Schuna, J., Johnson, W., Tudor-Locke, C., 2013. Adult self-reported and objectively monitored physical activity and sedentary behavior: NHANES 2005-2006. Int. J. Behav. Nutr. Phys. Act. 10, 126.

Shay, C.M., Ning, H., Allen, N.B., Carnethon, M.R., Chiuve, S.E., Greenlund, K.J., Daviglus, M.L., Lloyd-Jones, D.M., 2012. Status of cardiovascular health in US adults: prevalence estimates from the National Health and Nutrition Examination Surveys (NHANES) 2003-2008. Circulation 125, 45-56.

Sorlie, P.D. Aviles-Santa, L.M., Wassertheil-Smoller, S., Kaplan, R.C., Daviglus, M.L. Giachello, A.L., Schneiderman, N., Raij, L., Talavera, G., et al., 2010. Design and implementation of the Hispanic Community Health Study/Study of Latinos. Ann. Epidemiol. 20, 629-641.

Thompson, D., Batterham, A.M., Peacock, O.J., Western, M.J., Booso, R., 2016. Feedback from physical activity monitors is not compatible with current recommendations: a recalibration study. Prev. Med. 91, 389-394.

USDHHS, 2008. 2008 Physical Activity Guidelines for Americans. U.S. Department of Health and Human Services, Washington.

van de Kassteele, J., Hoogenveen, R.T., Engelfriet, P.M., Baal, P.H., Boshuizen, H.C., 2012. Estimating net transition probabilities from cross-sectional data with application to risk factors in chronic disease modeling. Stat. Med. 31, 533-543.

WHO, 2009. Global Health Risks, Mortality, and Burden of Disease Attributable to Selected Major Risks. World Health Organization, Geneva, Switzerland.

Willey, J.Z., Paik, M.C., Sacco, R., Elkind, M.S.V., Boden-Albala, B., 2010. Social determinants of physical inactivity in the Northern Manhattan Study (NOMAS). J. Community Health 35 (6), 602-608. 